\title{
Gefitinib induces premature senescence in non-small cell lung cancer cells with or without EGFR gene mutation
}

\author{
KATSUYUKI HOTTA, MASAHIRO TABATA, KATSUYUKI KIURA, TOSHIYUKI KOZUKI, \\ AKIKO HISAMOTO, HIDEKI KATAYAMA, NAGIO TAKIGAWA, NOBUKAZU FUJIMOTO, \\ KEIICHI FUJIWARA, HIROSHI UEOKA and MITSUNE TANIMOTO
}

Department of Medicine II, Okayama University Medical School, 2-5-1, Shikata-cho, Okayama 700-8558, Japan

Received May 23, 2006; Accepted July 24, 2006

\begin{abstract}
Despite its tremendous antitumor effect in a subset of patients with non-small cell lung cancer (NSCLC), the exact mechanism of gefitinib-induced cell death has not been fully determined. In this study, forms of cell death in various NSCLC cell lines after gefitinib exposure was analyzed to elucidate the cell death mechanism of gefitinib. Though higher concentration of gefitinib $(10 \mu \mathrm{M})$ induced extensive apoptosis in two cell lines (EGFR-mutated PC-9 cells and EGFR wildtype EBC-2/R cells), clinically relevant concentrations of gefitinib $(1 \mu \mathrm{M})$ induced prominent premature senescence instead of apoptosis in these cells. This induction of senescence was preceded by immediate increase of $\mathrm{p} 16^{\mathrm{INK} 4 \mathrm{~A}}$, p2 $1^{\text {WAF } 1 / \text { Cip } 1}$ and 2 $27^{\text {Kip } 1}$ levels and subsequent $G_{1}$ cell cycle arrest. These phenomena were not observed in gefitinibresistant (RERF-LC-MS) cells. Additionally, ex vivo exposure to gefitinib induced senescence in short-term cultured tumor cells that were obtained from malignant pleural effusion of a patient with NSCLC, whose tumor was later revealed to be clinically sensitive to gefitinib. Our results indicate that senescence might be a major anti-tumor mechanism of gefitinib in these NSCLC cells regardless of the EGFR gene mutation status.
\end{abstract}

\section{Introduction}

Gefitinib ('Iressa', ZD1839, a trademark of the AstraZeneca group of companies), an EGFR-tyrosine kinase inhibitor (1), has shown unprecedented anti-tumor activity in a subset of patients with non-small cell lung cancer (NSCLC) (2). However, the exact mechanism of gefitinib-induced cell death remains to be elucidated. Several studies have demonstrated that gefitinib-induced cell growth inhibition through apoptosis in various cancer cell lines including vulva, ovary,

Correspondence to: Dr Katsuyuki Hotta, Department of Medicine II, Okayama University Medical School, 2-5-1, Shikata-cho, Okayama 700-8558, Japan

E-mail:khotta@md.okayama-u.ac.jp

Key words: gefitinib, premature senescence, non-small cell lung cancer, apoptosis, EGFR colon and breast. However, in these studies, a higher concentration of gefitinib was required to induce apoptosis and the number of apoptotic cells was relatively small (12$20 \%$ ) (3-5). Even in the A431 human vulvar squamous cell carcinoma cell line, a representative cell line sensitive to gefitinib, only $10 \%$ of the cells developed apoptosis by gefitinib treatment (5). Therefore, apoptosis might not be a main mechanism of gefitinib-induced cell death. To explain clinically observed gefitinib-induced rapid and tremendous tumor regression, contribution of other additional mechanism of cell death was supposed. Senescence has been shown to play a central role in anti-tumor effect induced by different classes of anti-cancer agents and ionising radiation (6-8). In this study, we examined whether gefitinib could induce cellular senescence in gefitinib-sensitive NSCLC cells.

\section{Materials and methods}

Cell culture and reagents. Human NSCLC cell line PC-9 was obtained from Riken Bioresource Center (Tsukuba, Japan) and RERF-LC-MS was obtained from Japan Collection of Research Bioresources Cell Bank (Tokyo, Japan). EBC-2/R human NSCLC cell line was established in our institute (9). All cell lines were cultured in RPMI-1640 supplemented with $10 \% \mathrm{FBS}$ and incubated at $37^{\circ} \mathrm{C}$ in $5 \% \mathrm{CO}_{2}$. PC-9 and EBC$2 / \mathrm{R}$ are previously known to be sensitive to gefitinib and RERF-LC-MS is known to be resistant to gefitinib. Gefitinib was kindly provided by AstraZeneca Pharmaceuticals (Cheshire, UK; 'Iressa', is a trademark of the AstraZeneca group of companies). X-Gal and z-VAD-fmk was purchased from Sigma-Aldrich (St. Louis, MO) and Calbiochem (San Diego, CA). Gefitinib and z-VAD-fmk were made up in dimethylsulfoxide (DMSO) as stock solutions and stored at $-20^{\circ} \mathrm{C}$. Each stock solution was dissolved in fresh medium just before use. The final concentration of DMSO in the medium was adjusted to $1 \%$.

Nucleotide-sequence analysis. For mutational analysis of the EGFR coding sequence, DNA was extracted from each cell line using QIAamp DNA mini kit (Qiagen, Hilden, Germany) and exons 18, 19, 21 and 23 were amplified by the nested polymerase-chain reaction (PCR). Primer sequences and amplification conditions were described previously (10). PCR products were processed with the Big dye terminator 
cycle sequencing kit and analyzed in both sense and antisense directions for the presence of mutations on an ABI 3100 sequencer (both from Applied Biosystems, Foster City, CA).

Cellular assays. For growth assays, cells were seeded in 6-well plates at adequate number of cells/well. Twenty-four hours after plating, cells were placed in fresh media containing indicated concentrations of gefitinib, allowed to grow for 3-72 h and subsequently harvested. Number of viable cells was counted by the trypan blue exclusion test. Cell cycle distribution was analyzed by the FACSCalibur (Becton Dickinson, Mountain View, CA) using Cell Quest software after staining with propidium iodide. Flow cytometric analysis of apoptotis was performed by gating the hypodiploid region on the DNA content histogram. Also, apoptosis was morphologically determined after staining with both Hoechst 33342 and propidium iodide (Sigma-Aldrich) (11). Identification of senescence-like phenotype was performed using acidic Bgalactosidase (senescence-associated B-galactosidase, SA-BGal) staining (12).

Protein assays. Western blots were performed by harvesting total cellular lysates in $50 \mathrm{mM}$ Tris (pH 7.4), 1\% NP40, $0.25 \%$ $\mathrm{Na}$ deoxycholate, $150 \mathrm{mM} \mathrm{NaCl}$, and protease inhibitors. Each lysate was separated by SDS-PAGE, transferred to membrane, and immunoblotted using specific primary antibodies with enhanced chemiluminescence visualization. The following antibodies were used for Western blots: anti-EGFR (Cell Signaling Technology, Beverly, MA), anti-p53, -p16 ${ }^{\mathrm{INK} 4 \mathrm{~A}}$, -p21 WAF1/Cip1, and -p27 Kip1 (Santa Cruz Biotechnology, Santa Cruz, CA) and anti- $\beta$-actin (Sigma-Aldrich).

Ex vivo assays for apoptosis and senescence. Malignant pleural effusion was collected from a NSCLC patient before administration of gefitinib with fully informed consent. Isolated tumor cells were suspended in fresh media with or without gefitinib in a 6-well plate, allowed to grow for $72 \mathrm{~h}$, harvested subsequently and stained for apoptosis and senescence.

Statistical analysis. Statistical analysis was performed using the StatView 5.0 program (BrainPower Inc., Calabasas, CA). Mann-Whitney $\mathrm{U}$ test was used for group analysis and $\mathrm{p}$-values of $\leq 0.05$ were considered statistically significant.

\section{Results}

EGFR gene mutation analysis in NSCLC cells. Heterozygous in-flame 15-amino acid deletion within exon 19 of the EGFR gene (del E7460-A750) was observed in gefitinib-sensitive PC-9 cells. Gefitinib-sensitive EBC-2/R cells and gefitinibresistant RERF-LC-MS cells did not harbored any mutation in exons 18, 19, 21 and 23 of the EGFR gene.

Gefitinib induced cell growth inhibition without significant increase in apoptotic cell number in NSCLC cells. Clinically relevant concentrations of gefitinib $(0.1$ and $1 \mu \mathrm{M})$ induced significant growth inhibition in PC-9 and EBC-2/R cells, whereas even higher concentration of gefitinib $(10 \mu \mathrm{M})$ showed less growth inhibition in RERF-LC-MS cells (Fig. 1A).
A

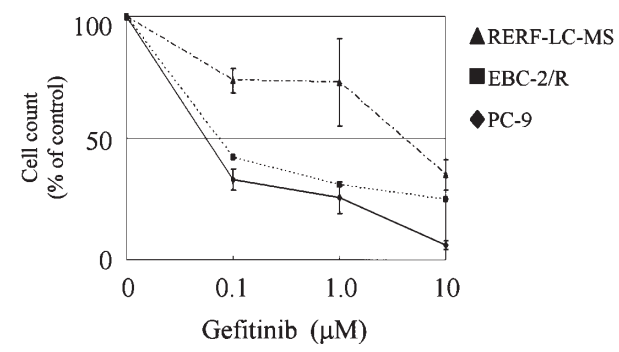

B
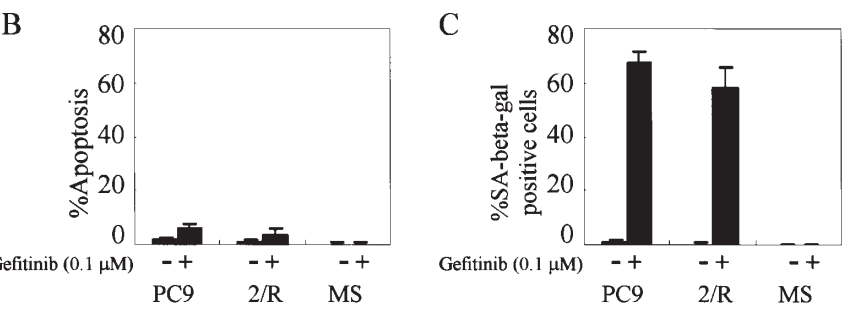

$\mathrm{D}$

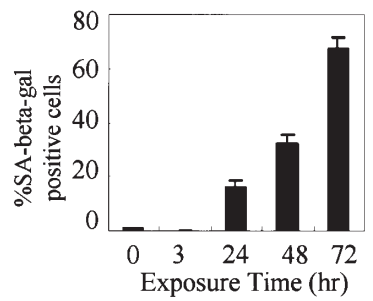

Figure 1. Gefitinib induces premature senescence in the gefitinib-sensitive cells. (A) Gefitinib induced cell growth inhibition in PC-9, EBC-2/R, and RERF-LC-MS cells. Cells were seeded in a 6-well plate and exposed to various concentrations of gefitinib for $72 \mathrm{~h}$, then trypsinized and counted. Results were average of quadruplicates. (B and C) Induction of apoptosis and senescence with gefitinib treatment in PC-9, EBC-2/R, and RERF-LCMS cells. Cells were seeded in a 6-well plate and treated with or without 0.1 $\mu \mathrm{M}$ of gefitinib for $72 \mathrm{~h}$. Then, cells were harvested and stained with Hoechst 33342 and propidium iodide to detect apoptosis, or fixed and stained with X-gal at pH 6.0 overnight to detect SA- $\beta$-Gal activity. Results were the average of triplicates. (D) Gefitinib induced premature senescence in PC-9 cells in a time-dependent manner. Cells were exposed to $0.1 \mu \mathrm{M}$ of gefitinib, then fixed and stained with X-gal at the indicated intervals. Results were the average of triplicates.

PC-9 cells, which are most sensitive to gefitinib among the three cell lines, showed $66.8 \pm 6.9 \%$ growth inhibition after $72 \mathrm{~h}$ continuous exposure to $0.1 \mu \mathrm{M}$ of gefitinib; however, at this point, only $6.3 \pm 0.9 \%$ cells showed apoptosis by Hoechst 33342 staining (Fig. 2A). Number of gefitinib-induced apoptotic cells was increased in a dose-dependent manner and the apoptotic cells increased up to $72.7 \pm 2.6 \%$ with a higher concentration of gefitinib $(10 \mu \mathrm{M})$, although this concentration is clinically not achievable. Another gefitinibsensitive cell line, EBC-2/R, showed similar results, whereas gefitinib-resistant RERF-LC-MS cells showed far less apoptotic cells with even a higher concentration of gefitinib $(10 \mu \mathrm{M})$ (Fig. 1B). By flow cytometry, PC-9 cells showed immediate $\mathrm{G}_{1}$ cell cycle accumulation as early as $3 \mathrm{~h}$ after exposure to even the lowest dose of gefitinib $(0.1 \mu \mathrm{M})$. However, cells in hypodiploid region, which is considered to represent apoptotic cells, was not remarkable even after $72 \mathrm{~h}$ continuous exposure to gefitinib (Fig. 3A). 

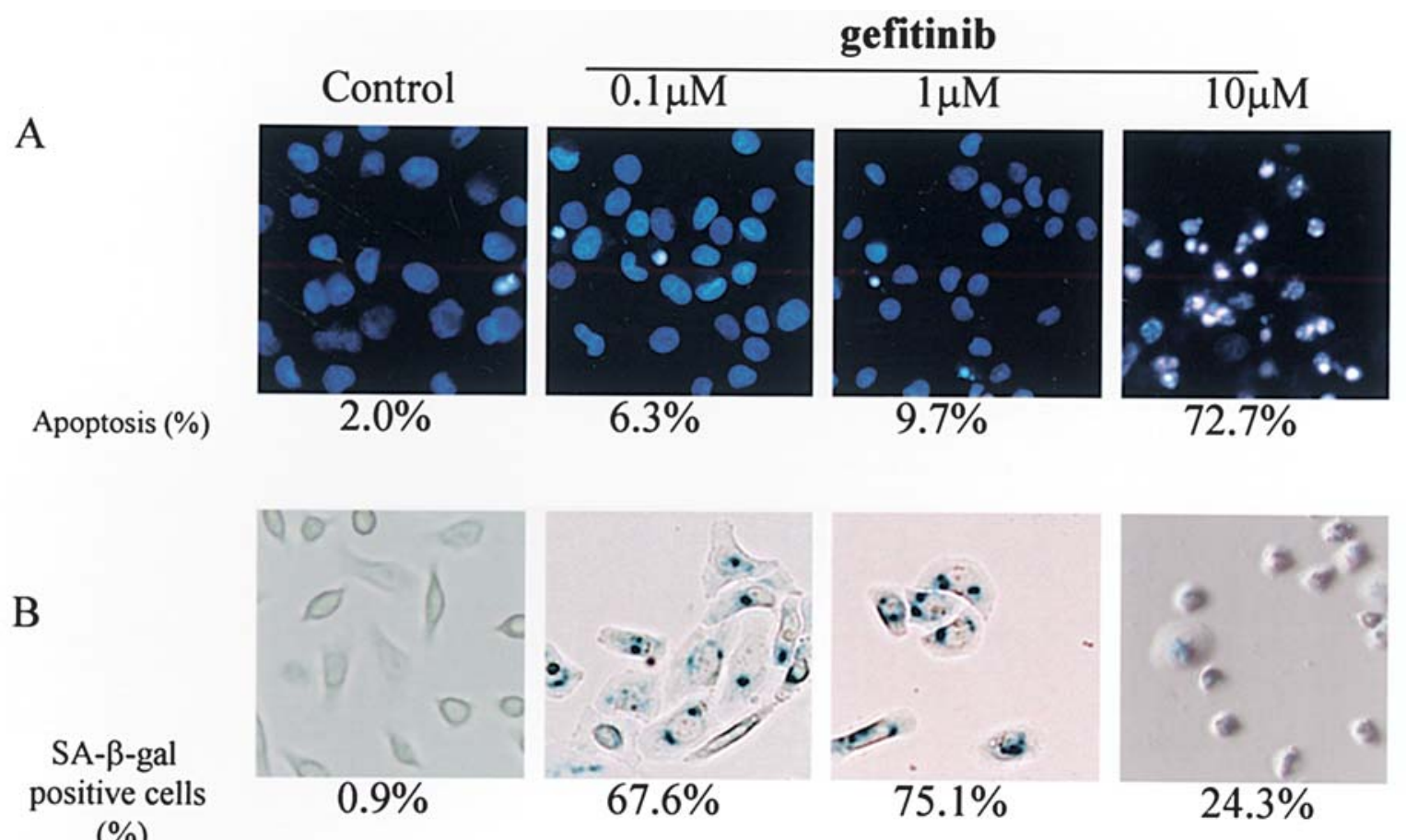

Figure 2. Gefitinib induces premature senescence in the gefitinib-sensitive cells. Exponentially growing PC-9 cells were exposed to indicated concentrations of gefitinib for $72 \mathrm{~h}$ and subsequently harvested. (A) Cells were stained with Hoechst 33342 and propidium iodide, and apoptosis was analyzed under a fluorescent microscopy (x400). Apoptotic cells showed condensation of chromatin and nuclear fragmentation. (B) Cells were fixed and incubated with X-gal at $\mathrm{pH} 6.0$ overnight and senescence-like phenotype was analyzed under microscopy (x400). Senescence-like cell death was predominant with clinically relevant concentrations of gefitinib; however, apoptotic cell death was predominant with very high concentration of gefitinib.

Gefitinib induces premature senescence instead of apoptosis in NSCLC cells. Gefitinib-sensitive cells showed $\mathrm{G}_{1}$ arrest without increase of apoptosis by gefitinib treatment. The observation that these gefitinib-treated cells showed enlarged and flattened morphology suggested an induction of senescence in these cells. Indeed, in PC-9 cells, SA-ß-Gal positive cells appeared at $24 \mathrm{~h}$ after treatment with clinically relevant concentration of gefitinib and the number of SA-B-Gal positive cells increased extensively in a time- and dose-dependent manner $(67.6 \pm 3.9 \%$ and $75.1 \pm 3.0 \%$ at $72 \mathrm{~h}$ after 0.1 and $1 \mu \mathrm{M}$ of gefitinib treatment, respectively) (Figs. 1D and 2B); however, number of SA-B-Gal positive cells remarkably decreased $(24.3 \pm 0.3 \%)$ and apoptotic cells became dominant $(72.7 \pm 2.6 \%)$ with highest concentration of gefitinib $(10 \mu \mathrm{M})$ (Fig. 2A). Also, gefitinib induced significant increase of SAB-Gal positive cells in EBC-2/R with clinically relevant concentration of gefitinib (Fig. 1C). In contrast, gefitinib induced no SA-ß-Gal positive cells in gefitinib-resistant RERF-LCMS cells. Thus, the gefitinib-induced growth inhibition in PC-9 (mutated EGFR) and EBC-2/R (wild-type EGFR) was considered to be characterized mainly by induction of premature senescence rather than apoptosis. To confirm this, we studied whether the cell growth inhibition by gefitinib could be abrogated by inhibition of apoptosis or not. Inhibition of the apoptotic pathway by caspase-3 inhibitor z-VAD-fmk $(50 \mu \mathrm{M})$ prevented PC-9 cells from developing apoptosis after treatment with $0.1 \mu \mathrm{M}$ of gefitinib $(1.0 \pm 0.6 \%$ vs. $6.3 \pm 0.9 \%$ with and without $\mathrm{z}-\mathrm{VAD}-\mathrm{fmk}$, respectively, $\mathrm{p}=0.050)$. Although apoptosis was significantly inhibited, gefitinib induced cell growth inhibition was not affected by
z-VAD-fmk treatment $(69.5 \pm 1.7 \%$ vs. $67.7 \pm 7.7 \%$ with and without $\mathrm{z}-\mathrm{VAD}-\mathrm{fmk}$, respectively, $\mathrm{p}=0.513$ ). Number of SA$\beta$-Gal positive cells was also identical irrespective of $\mathrm{z}$-VADfmk treatment $(61.6 \pm 1.2 \%$ vs. $67.2 \pm 3.1 \%$ with and without Z-VAD-fmk, respectively, $\mathrm{p}=0.127$ ).

Gefitinib treatment resulted in immediate and temporal upregulation of $C D K$ inhibitors. Generally, induction of senescence is defined by up-regulation of CDK-inhibitors and activation of p53 (6-8). In PC-9 cells, immediate and temporal up-regulation of $\mathrm{p} 21^{\mathrm{WAF} 1 / \mathrm{Cip} 1}$ followed by temporal up-regulation of $\mathrm{p} 27^{\mathrm{Kip} 1}$ was also observed with gefitinib treatment (Fig. 3B). Up-regulation of $\mathrm{p} 21^{\mathrm{WAF} 1 / \mathrm{Cip} 1}$ was observed as early as $3 \mathrm{~h}$ after gefitinib treatment, when cells were going to accumulate in $\mathrm{G}_{1}$ cell cycle $(56.3 \pm 0.9 \%$ vs. $72.8 \pm 1.1 \%$, before and $3 \mathrm{~h}$ after treatment with $0.1 \mu \mathrm{M}$ of gefitinib, respectively). This up-regulation of CDK inhibitors was not observed in gefitinib-resistant RERF-LC-MS cells. Both cell lines harbored mutated p53 $(13,14)$ and expression of p53 protein was not altered by treatment with gefitinib in either cell line (Fig. 3B).

Gefitinib induce premature senescence in human NSCLC tumor ex vivo. To investigate whether gefitinib induces premature senescence in a human tumor or not, SA- $3-$ Gal assay was done using short-term cultured tumor cells collected from malignant pleural effusion in a 73-year-old man with locally advanced NSCLC. Cytologically, numerous tumor clusters were observed in the effusion (Fig. 4A). After $72 \mathrm{~h}$ gefitinib exposure ex vivo, significant increase of SA- $\beta-G a l$ positive 


\section{A}
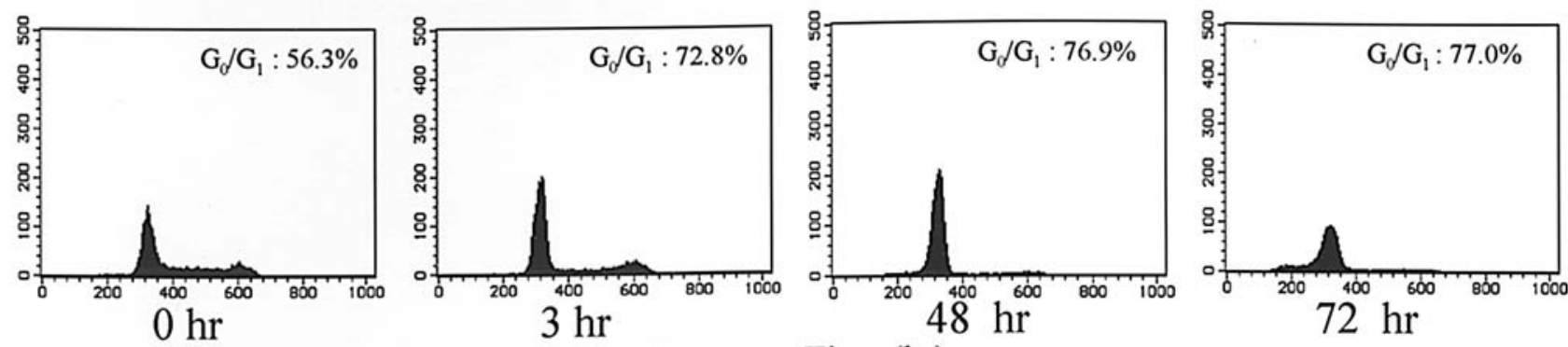

Exposure Time (hr)

$\mathrm{B}$

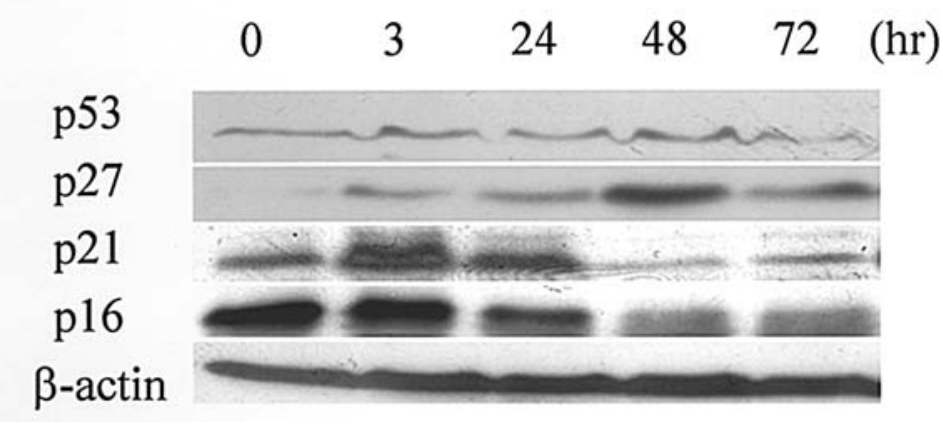

Figure 3. (A) Gefitinib induced $\mathrm{G}_{1}$ cell cycle arrest in PC-9 cells. Cells were treated with $0.1 \mu \mathrm{M}$ of gefitinib, harvested at the indicated intervals, stained with propidium iodide and analyzed by flow cytomety. (B) Gefitinib induced immediate up-regulation in CDK inhibitors. Cells were treated with $0.1 \mu \mathrm{M}$ of gefitinib for indicated periods. Then, cellular lysates were harvested, separated by SDS-PAGE, and immunoblotted with indicated antibodies.
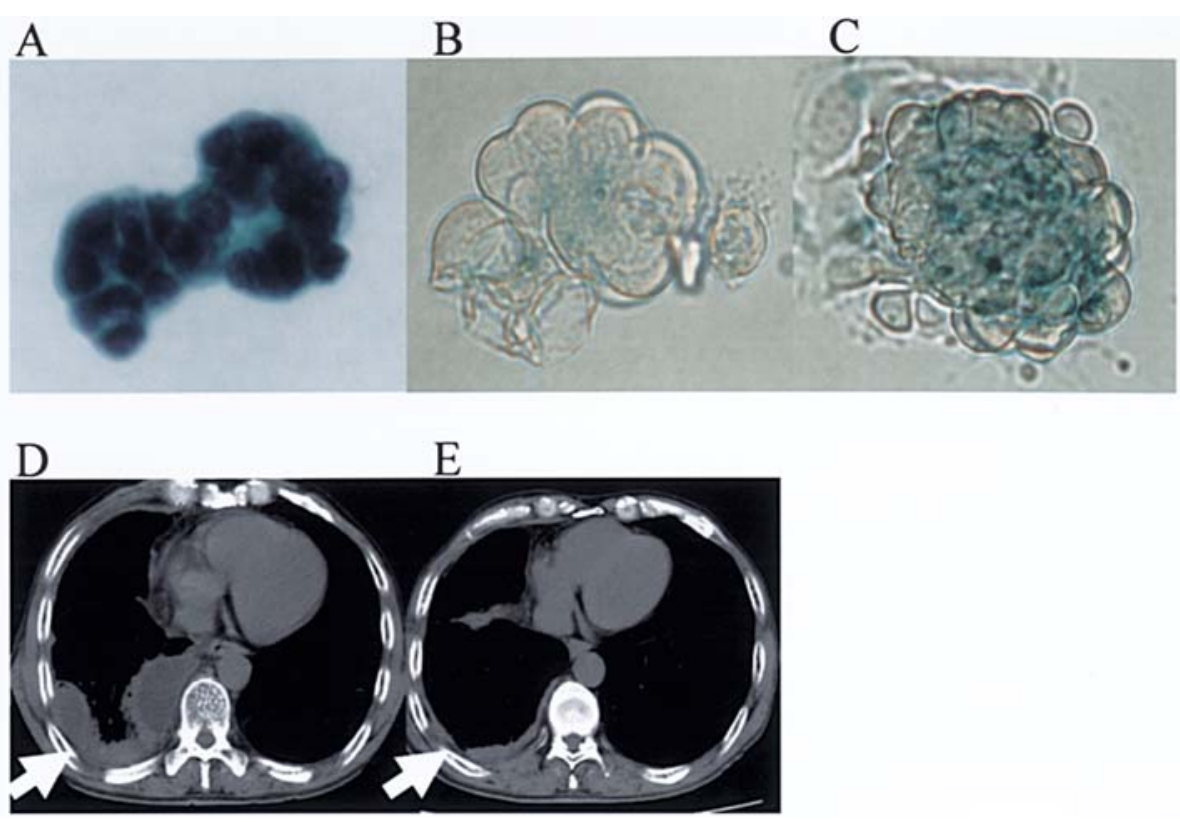

Figure 4. Gefitinib induced premature senescence in a human NSCLC tumor ex vivo. Tumor cells were collected from malignant pleural effusion in a NSCLC patient before administration of gefitinib. (A) Isolated tumor cells (Papanicoleau stain, $\mathrm{x} 400$ ). (B and C) Collected tumor cells were suspended in fresh media with $\mathrm{C}$, or without $\mathrm{B}, 0.1 \mu \mathrm{M}$ of gefitinib in a 6-well plate, and allowed to grow for $72 \mathrm{~h}$, harvested subsequently and stained with X-gal at $\mathrm{pH} 6.0$ overnight to detect SA-ß-Gal activity (x400). (D and E) Computed tomographic scans of chest before (D), and 15 days after (E), daily gefitinib treatment (250 mg/day).

cells was observed with clinically relevant concentrations $(0.1 \mu \mathrm{M})$ of gefitinib, whereas an increase in apoptosis was not observed (Fig. 4B and C). In accordance with this ex vivo result, the patient obtained a clinically objective response with daily oral administration of gefitinib (250 mg/day). His pleural effusion was markedly diminished on day 15 (Fig. 4D 
and E) accompanied with improvement of clinical symptoms such as dyspnea on effort and cough.

\section{Discussion}

This study shows that clinically relevant concentrations of gefitinib induced $G_{1}$ cell cycle accumulation and increase of SA-ß-Gal positive cells, which are considered indicative of senescent cells in two gefitinib-sensitive NSCLC cell lines and one clinical specimen. Replicative senescence is an essential process of aging and occurs following DNA damaging response activated by the shortening of telomeres. The DNA damage response triggered by other cellular stresses, including exposure to chemotherapeutic agents can also induce senescence, often termed premature senescence (15-17). Cisplatin, hydroxyurea, irinotecan, daunorubicin and retinoic acid have also been reported to induce senescence in various tumor cell lines $(6-8,18)$. Although gefitinib is not known as a DNA damaging agent, it might be possible that gefitinib modulated the repair of DNA by involving the DNA-dependent protein kinase (DNA-PK) pathway (19). The molecular signals that play critical roles in mediating DNA damage-induced scenescence include p53, CDK inhibitors (Rb, p16 $\left.6^{\mathrm{INK} 4 \mathrm{~A}}, \mathrm{p} 21^{\mathrm{WAF} / \mathrm{Cip} 1}\right)$, and Bcl-2 (6). In this study, gefitinib treatment led to a rapid and temporal up-regulation of $\mathrm{p} 21^{\mathrm{WAF} / \mathrm{Cip} 1}$ and $\mathrm{p} 27^{\mathrm{Kip} 1}$, and subsequent $\mathrm{G}_{1}$ cell cycle accumulation before emergence of SA-ß-Gal positive cells. In PC-9 cells, gefitinib treatment did not induce the up-regulation of $\mathrm{p} 16^{\mathrm{INK} 4 \mathrm{~A}}$. It seems to be that induction of $\mathrm{p} 21^{\mathrm{WAF} / \mathrm{Cip} 1}$ rather than $\mathrm{p} 16^{\mathrm{INK} 4 \mathrm{~A}}$ dominantly led to $\mathrm{G}_{1}$ arrest in this cell line, as reported by Mansilla et al and te Poele et al $(7,18)$.

There is also considerable interest in defining predictors for clinical response to gefitinib. Lynch et al showed that specific mutations in the EGFR gene correlated with clinical responsiveness to gefitinib and concluded that screening for EGFR mutations in NSCLC might identify patients who will have a response to gefitinib (10). We demonstrated that only one of the two gefitinib-sensitive cells had in-flame deletion within exon 19, which suggests that gefitinib-induced growth inhibition and induction of premature senescence are not always associated with the EGFR mutation. Further investigation is warranted to clarify the relationship between these mutations and induction of senescence and cell growth inhibition.

Nagourney et al showed that the results of gefitinib sensitivity analysis by MTT assay using short-term cultured human tumors correlated well with clinical outcome in NSCLC patients treated with gefitinib (20). In our study, we observed induction of senescence-like phenotype in short-term cultured tumor cells from a patient who showed successful response to gefitinib. Based on these results, ex vivo evaluation of the gefitinib activity using SA- $\beta-$ Gal staining of short-term cultured tumor cells obtained from patients might enable us to predict the clinical response to gefitinib.

In conclusion, we are first to demonstrate that induction of premature senescence might be a mechanism of tumor cell kill of EGFR tyrosine kinase inhibitor, gefitinib. Since our findings were based on experiments using a limited number of cell lines and a clinical specimen, future confirmatory study should be planned. If confirmed, elucidation of the factors that regulate treatment-induced senescence should be useful in improving the efficacy of cancer therapy.

\section{References}

1. Woodburn JR: The epidermal growth factor receptor and its inhibition in cancer therapy. Pharmacol Ther 82: 241-250, 1999.

2. Fukuoka M, Yano S, Giaccone G, et al: Multi-institutional randomized phase II trial of gefitinib for previously treated patients with advanced non-small cell lung cancer. J Clin Oncol 21: 2237-2246, 2003

3. Gilmore AP, Valentijn AJ, Wang P, et al: Activation of BAD by therapeutic inhibition of epidermal growth factor receptor and transactivation by insulin-like growth factor receptor. J Biol Chem 277: 27643-27650, 2002.

4. Anido J, Matar P, Albanell J, et al: ZD1839, a specific epidermal growth factor receptor (EGFR) tyrosine kinase inhibitor, induces the formation of inactive EGFR/HER2 and EGFR/ HER3 heterodimers and prevents hergulin signaling in HER2overexpressing breast cancer cells. Clin Cancer Res 9: 1274-1283, 2003.

5. Janmaat ML, Kruyt FAE, Rodriguez JA and Giaccone G: Response to epidermal growth factor inhibitors in non-small cell lung cancer cells: limited antiproliferative effects and absence of apoptosis associated with persistent activity of extracellular signal-regulated kinase or Akt kinase pathways. Clin Cancer Res 9: 2316-2326, 2003.

6. Wainwright LJ, Lasorella A and Lavarone A: Distinct mechanisms of cell cycle arrest control the decision between differentiation and senescence in human neuroblastoma cells. Proc Natl Acad Sci USA 98: 9396-9400, 2001.

7. Mansilla S, Pina B and Portugal J: Daunorubicin-induced variations in gene transcription: commitment to proliferation arrest, senescence and apoptosis. Biochem J 372: 703-711, 2003.

8. Han Z, Wei W, Dunaway S, et al: Role of p21 in apoptosis and senescence of human colon cancer cells treated with camptothecin. J Biol Chem 277: 17154-17160, 2002.

9. Kawai H, Kiura K, Tabata M, et al: Characterization of non-small cell lung cancer cell lines established before and after chemotherapy. Lung Cancer 35: 305-314, 2002.

10. Lynch TJ, Bell DW, Sordella R, et al: Activating mutations in the epidermal growth factor receptor underlying responsiveness of non-small cell lung cancer to gefitinib. N Engl J Med 350: 2129-2139, 2004

11. Tabata M, Tabata R, Grabowski DR, Bukowski RM, Ganapathi MK and Ganapathi R: Roles of NF-kappaB and 26 S proteasome in apoptotic cell death induced by topoisomerase I and II poisons in human non-small cell lung carcinoma. J Biol Chem 276: 8029-8036, 2001.

12. Dimri GP, Lee X, Basile G, et al: A biomarker that identifies senescent human cells in culture and in aging skin in vivo. Proc Natl Acad Sci USA 92: 9363-9367, 1995.

13. Ookawa K, Kudo T, Aizawa S, Saito H and Tsuchida S: Transcriptional activation of the MUC2 gene by p53. J Biol Chem 277: 48270-48275, 2002.

14. Yano T, Sugio K, Yamazaki K, et al: Direct IFNluence of interferon-gamma on proliferation and cell-surface antigen expression of non-small cell lung cancer cells. Lung Cancer 30: 169-174, 2000.

15. Schmitt CA, Fridman JS, Yang M, et al: A senescence program controlled by $\mathrm{p} 53$ and $\mathrm{p} 16^{\mathrm{INK} 4 \mathrm{~A}}$ contributes to the outcome of cancer therapy. Cell 109: 335-346, 2002.

16. Robles SJ and Adami GR: Agents that cause DNA double strand breaks lead to $\mathrm{p} 16^{\mathrm{INK} 4 \mathrm{~A}}$ enrichment and the premature senescence of normal fibroblasts. Oncogene 16: 1113-1123, 1998.

17. Ricci MS and Zong WX: Chemotherapeutic approaches for targeting cell death pathways. Oncologist 11: 342-357, 2006.

18. te Poele RH, Okorokov AL, Jardine L, Cummings J and Joel SP: DNA damage is able to induce senescence in tumor cells in vitro and in vivo. Cancer Res 62: 1876-1883, 2002.

19. Friedmann BJ, Caplin M, Savic B, et al: Interaction of the epidermal growth factor receptor and the DNA-dependent protein kinase pathway following gefitinib treatment. Mol Cancer Ther 5: 209-218, 2006.

20. Nagourney R, Chow C, Kollin C, Su YZ, Horlick D and Evans S Effect of gefitinib ('Iressa', ZD1839) in human tumor primary cultures: possible insights into drug actions. Proc Am Assoc Cancer Res 44: 757, 2003. 